\title{
Über den Molekularzustand von geschmolzenem Chlorsilber.
}

Von Richard lorenz, A. Liebmann und A. Höchberg.

Über den Molekularstand einer Reihe von geschmolzenen Salzen ist schon früher berichtet worden. ${ }^{1}$ Es war hierbei durch Messung der Oberflächenspannung an flüssigen Schmelzen und Anwendung der Regel von Eörvös gezeigt worden, daß in den Salzschmelzen auf Assoziation geschlossen werden muß̈, da die Konstante der molaren Oberflächenenergie wesentlich kleiner gefunclen ist als der von Ramsay und SHIElds für die „normalen Flüssigkeiten“ ermittelte Wert. Linbuans, der die Messungen der Oberflächenspannung in ihrer Abhängigkeit von der Temperatur durchgeführt hat ${ }^{2}$, bestimmte diese Größe auch an geschmolzenem Chlorsilber; doch konnten die erhaltenen Ergebnisse für die Kenntnjs des Molekularzustandes dieses Stoffes nicht verwertet werden, da die Dichten lesselben unbekannt waren. Durch die diesbezüglichen kürzlich reröffentlichten Bestimmungen von A. HöchBeRG ${ }^{3}$ ist es jetzt möglich geworden, die Limbmannschen Ergebnisse zu berechnen. Die Meßmethode beruht auf der Messung der Steighöhe in Kapillaren bei verschiedenen Temperaturen. Bezeichnet $h$ die Steighöhendifferenz zwischen dem Flüssigkeitsspiegel in der Kapillare und dem in dem weiten Rohransatz $r_{1}$ den Halbmesser der Kapillare, $r_{\varepsilon}$ den Halbmesser der weiten Röhre, $s$ die Dichte des Stoffes bei der entsprechenden Temperatur, $a$ den Ausdehnungskoeftizienten des Glases, so berechnet sich die Oberflächenspannung der Flüssigkeit nach der Formel:

$$
x=\frac{s h(1+a t)}{2\left(\frac{1}{x_{1}}-\frac{1}{r_{2}}\right)} \mathrm{g} / \mathrm{cm} .
$$

1 Richard Lorenz und F. Kaufler, Der Molekularzustand der geschmolzenen Salze. Ber. d. d. chem. Ges. 41 (1908). 3727.

2 A. Liebmann, Zur Kenntnis der Oberflächenspanmung geschmolzener Metalle und Salze. Inaug.-Dissert. Zürich 1909.

3 A. HöchBkra, Physikalisch-chemische Messungen an geschmolzenen Silberhaloiden. Inaug.-Dissert. Frankfurt a. M. 1915; siehe auch Rich. Loranz und A. HöchBerg, Zeitschr. f. anorg. u. allg. Chem. $9 \pm$ (1916) 288. 
Nachstehende Tabelle zeigt die für $x$ nach obiger Formel berechneten Werte. Unter $h$ stehen die von LIEBMans beobachteten Steighöhendifferenzen, unter $s$ die nach der Interpolationsformel von Höchberg für die Dichte berechneten Werte.

\begin{tabular}{|c|c|c|c|c|c|}
\hline Temp. & $h$ & $s$ & $(1+a)$ & $x \mathrm{~g} / \mathrm{cm}$ & $x \mathrm{Dyn} / \mathrm{cm}$ \\
\hline 582 & 0,785 & 4,732 & 1,0033 & 0,1158 & 113.6 \\
\hline 579 & 795 & 4.735 & 33 & 1168 & 114.6 \\
\hline 576 & 805 & 4.737 & 33 & 1183 & 116.1 \\
\hline 573 & 780 & 4.740 & 33 & 1150 & 112.8 \\
\hline 568 & 785 & 4.744 & 33 & 1156 & 113.4 \\
\hline 562 & 780 & 4.750 & 33 & 1150 & 112.8 \\
\hline 558 & 790 & 4.754 & 33 & 1165 & 114.3 \\
\hline 554 & 785 & 4.757 & 33 & 1159 & 113.7 \\
\hline 550 & 775 & 4.761 & 33 & 1145 & 112.3 \\
\hline 546 & 790 & 4.765 & 33 & 1168 & 114.6 \\
\hline 541 & 800 & 4.769 & 30 & 1184 & 116.2 \\
\hline 536 & 790 & 4.774 & 30 & 1170 & 114.8 \\
\hline 532 & 800 & 4.777 & 30 & 1186 & 116.3 \\
\hline 527 & 810 & 4.782 & 30 & 1202 & 117.9 \\
\hline 523 & 790 & 4.786 & 30 & 1173 & 115.1 \\
\hline 517 & 820 & 4.791 & 30 & 1219 & 119.6 \\
\hline 513 & 795 & 4.795 & 30 & 1183 & 116.0 \\
\hline 507 & 825 & 4.801 & 30 & 1229 & 120.5 \\
\hline 503 & 790 & 4.804 & 30 & 1177 & 115.5 \\
\hline 494 & 830 & 4.812 & 30 & 1239 & 121.6 \\
\hline 488 & 835 & 4.818 & 30 & 1248 & 122.4 \\
\hline 483 & 840 & 4.823 & 30 & 1257 & 120.5 \\
\hline 480 & 830 & 4.825 & 30 & 1243 & 121.9 \\
\hline 476 & 835 & 4.829 & 30 & 1251 & 122.7 \\
\hline 472 & 840 & 4.833 & 27 & 1260 & 123.6 \\
\hline 468 & 845 & 4.836 & 27 & 1267 & 124.3 \\
\hline 465 & 850 & 4.839 & 27 & 1276 & 125.2 \\
\hline 463 & 850 & 4.841 & 27 & 1276 & 125.2 \\
\hline 460 & 845 & 4.844 & 27 & 1270 & 124.5 \\
\hline 456 & 847 & 4.847 & 27 & 1270 & 124.5 \\
\hline 454 & 855 & 4.849 & 27 & 1286 & 125.9 \\
\hline 452 & 850 & 4.852 & 27 & 1279 & 125.5 \\
\hline 449 & 855 & 4.854 & 27 & 1287 & 126.3 \\
\hline 447 & 845 & 4.856 & 27 & 1273 & 124.9 \\
\hline
\end{tabular}

Es ist hierbei zu beachten, dak die Interpolationsformoln für liv Dichte streng genommen nur für das gemessene Temperaturintervall Geltung haben. Doch ist anzunehmen, daß die Kurven, die die Dichten der geschmolzenen Salze in ihrer Abhängigkeit von 
der Temperatur bezeichnen, bis zum Schmelzpunkt herab annähernd greradlinig verlaufen. Die Formeln dürfen daher wohl, wie in diesem Falle, auch mit guter Annäherung für die Berechnung tiefer liegender Punkte gebraucht werden.

Aus den so erhaltenen Werten für die Oberflächenspannung läBt sich nun init Hilfe der Formel von Förvös

$$
k=\frac{d}{d t} x(M v)^{3}=\frac{x_{1}\left(\frac{M}{s_{1}}\right)^{\frac{2}{3}}-x_{2}\left(\frac{M}{s_{2}}\right)^{\frac{2}{3}}}{t_{1}-t_{2}}
$$

die Konstante $k$ ermitteln. Für nicht assoziierte Flüssigkeiten hat diese Konstante bekanntlich nach Ramsay und SHIELds den ungefähren Wert von 2.12. Bekanntlich ist es wegen der ungenügenden theoretischen Begründung des Gesetzes von Eörvös nicht angängig, aus den gefundenen Größen von $k$ und der Eötvös-RAmsay-SHIELDSschen Zahl den ,Assoziationstabtor" genau zu bestimmen. Immerhin läBt sich durch einen Vergleich der beiden Zahlen eine ungefähre Angabe über die Größe einer etwaigen Assoziation erreichen. Aus der obigen Tabelle berechnet sich

$$
k=0.8
$$

Man num daraus auf eine starke Assoziation des Chlorsilbers schließen. Es werden sich durchschnittlich ungefähr 3-5 Moleküle aneinanderlagern. Diese Feststellung ist für die Theorie des Dissoziationsgrades und der Leitfähigkeit der geschmolzenen Salze von erheblichem Interesse. In bezug auf das Bromsilber und das Jodsilber liegt leider kein Beobachtungsmaterial vor; doch ist anzunehmen, daf, hier, wie bei so vielen Eigenschaften, weitgehende Analogie mit dem Chlorsilber besteht.

\section{Zusammenfassung.}

1. Die Oberflächenspannung von geschmolzenem Chlorsilber: wurde in dem Temperaturintervall von $447-582^{\circ} \mathrm{C}$ bestimmt, und mittels der Formel von Eörvös hieraus die RamsaY-SHIeldssche Konstante der molekularen Oberflächenenergie berechnet. 
304 Lorenz, Liebmann u. Höchberg. Molekularzustand v. geschm. Chlorsilber.

2. Die Konstante ergab sich zu

$$
k=0,8
$$

anstatt 2.12, wie sie nach RAMsAy und SHreLds den ,,normalen Flüssigkeiten" eigen ist. Geschmolzenes Chlorsilber dürfte daher als außerordentlich stark assoziiert angesehen werden können.

Frankfurt a. M., Institut für physikalische Chemie der Universität und des Physikalischen Vereins. Oktober 1915.

Bei der Redaktion eingegangen am 8. Dezember 1915. 